\title{
Gravitational lensing statistical properties in general FRW cosmologies with dark energy component(s): analytic results
}

\author{
Zong-Hong Zhu* \\ Beijing Astronomical Observatory, Chinese Academy of Sciences, Beijing 100012, China \\ National Astronomical Observatories, Chinese Academy of Sciences, Beijing 100012, China
}

\begin{abstract}
Various astronomical observations have been consistently making a strong case for the existence of a component of dark energy with negative pressure in the universe. It is now necessary to take the dark energy component(s) into account in gravitational lensing statistics and other cosmological tests. By using the comoving distance we derive analytic but simple expressions for the optical depth of multiple image, the expected value of image separation and the probability distribution of image separation caused by an assemble of singular isothermal spheres in general FRW cosmological models with dark energy component(s). We also present the kinematical and dynamical properties of these kinds of cosmological models and calculate the age of the universe and the distance measures, which are often used in classical cosmological tests. In some cases we are able to give formulae that are simpler than those found elsewhere in the literature, which could make the cosmological tests for dark energy component(s) more convenient.
\end{abstract}




\section{INTRODUCTION}

The Hubble expansion, the Cosmic Microwave Background Radiation(CMBR) and the primordial Big Bang Nucleosynthesis(BBN) are three cornerstones of the standard hot Big Bang cosmological model. Recently these three kinds of observations have been making a strong case for the existence of a nearly uniform component of dark energy with negative pressure: More than twenty experiments of CMBR measurements have now covered three orders of magnitude in multipole number, and are beginning to define the position of the first acoustic peak at a value that is consistent with a flat universe [1]; However, the deuterium abundance measured in four high redshift hydrogen clouds seen in absorption against distant quasars [2] combined with baryon fraction in galaxy clusters from X-ray data [3] give a low matter density universe, $\Omega_{m} \sim 0.3-0.4$; The discrepancy between the low matter density and a flat universe is observationally resolved by high redshift Ia type supernovae (SNeIa) observations [4], which implies an accelerating universe driven by a dark, exotic form of energy component. For a recent review of observational evidence for the dark energy component, reader is referred to Ref. [5]. It seems that determining the amount and nature of the dark energy is emerging as one of the most important challenges in cosmology.

The simplest possibility for the dark energy component is cosmological constant $\Lambda$ or equivalently vacuum energy [6]. In the past, a nonzero cosmological constant has been advocated and then with the improved observational data discarded several times [7]. Due to this checked history and the difficulty in understanding the observed $\Lambda$ in the framework of modern quantum field theory, now most physicists and astronomers prefer other candidates for dark energy, including a frustrated network of topological defects such as strings or walls [8] and an evolving scalar field referred to by some as quintessence [9] etc. As shown in literatures, it is difficult to discriminate against these different possibilities either by the SNeIa data alone [10 or only by the CMBR data [11]. This led some authors to consider the combination of the SNeIa measurements with the anisotropy of CMBR [12 or the large scale structures 13. 
Because of the potential importance of probing the dark energy component and uncertainties of present constraints, it is worthy of examining various cosmological tests for dark energy component(s). It has been long shown that gravitational lensing statistics is an efficient tool for determining cosmological parameters [14,15]. Some authors have even given the general expressions for the optical depth and average image separation in general FriedmanRobertson-Walker (FRW) cosmological models [16]. But these expressions are complicated and thereby hard to apply in practice. Furthermore, they didn't involve general dark energy component(s). We reconsider here gravitational lensing statistical properties, such as the optical depth of multiple image, the expectation value of image separation and the probability distribution of image separation, in general FRW cosmologies with various matter and energy components. We also calculate the age of the universe and distance measures, such as the luminosity distance and the angular diameter distance, which are often involved in classical cosmological tests. In some cases we are able to give formulae that are simpler than those found elsewhere in the literature, which could make the cosmological tests for dark energy component(s) more convenient.

The paper is organised as follows: In section 2, we summarize kinematics and dynamics of general FRW cosmologies with dark energy component(s); In section 3, we derive expressions for the age of the universe and the various distance measures; In section 4, using the comoving distance, we generalize and simplify lensing formulae; Results and discussion are given in section 5 .

\section{KINEMATICS AND DYNAMICS OF GENERAL FRW COSMOLOGIES WITH DARK ENERGY COMPONENT(S)}

For general FRW cosmologies, the metric of spacetime is described by (in the $c=1$ unit):

$$
d s^{2}=-d t^{2}+R^{2}(t)\left[d \chi^{2}+f^{2}(\chi)\left(d \theta^{2}+\sin ^{2} \theta d \phi^{2}\right)\right]
$$

where $f(\chi)=\chi$ for a flat universe $(k=0), f(\chi)=\sin \chi$ for a closed universe $(k=+1)$, and $f(\chi)=\sinh \chi$ for an open universe $(k=-1)$. Defining the scale factor $a(t)=R(t) / R_{0}$ 
$a=1$ today, and the Friedmann equation takes the form

$$
\begin{aligned}
\left(\frac{\dot{a}}{a}\right)^{2} & =\frac{8 \pi G}{3} \sum_{i} \rho_{i}-\frac{k}{a^{2} R_{0}^{2}} \\
\frac{\ddot{a}}{a} & =-\frac{4 \pi G}{3} \sum_{i}\left(\rho_{i}+3 p_{i}\right)
\end{aligned}
$$

where the dot represents derivatives with respect to $t$, and $i$ includes all components of matter or energy in the universe, e.g., $i=m$ for the total nonrelativistic matter, $r$ for the total radiation component, $\Lambda$ for the cosmological constant $\left(\rho_{\Lambda} \equiv \Lambda /(8 \pi G)\right), c$ for the cold dark matter, $h$ for the hot dark matter, $b$ for the baryons and $x$ for some unknown exotic component. If the effective equation of state for the $i$-th component is parameterized as $\omega_{i}=p_{i} / \rho_{i}$, its density scales as $\rho_{i} \propto a^{-n_{i}}$ where $n_{i}=3\left(1+\omega_{i}\right)$. For instance, nonrelativistic matter scales as $\rho_{m} \propto a^{-3}$ while relativistic matter, such as radiation, changes as $\rho_{r} \propto a^{-4}$, and vacuum energy(cosmological constant) is invariant $\left(\rho_{\Lambda} \propto a^{0}\right)$ as the universe expands. Eq.(2) tells us that all components of matter or energy and a curvature term conspire to

drive the universal expansion. It is convenient to assign symbols to their respective fractional contributions at the present epoch. Defining parameters

$$
\Omega_{i}=\frac{8 \pi G}{3 H_{0}^{2}} \rho_{i 0}, \quad \Omega_{k}=\frac{-k}{R_{0}^{2} H_{0}^{2}},
$$

where $H_{0}$ is the Hubble constant and zero subscripts refer to the present epoch. Eq.(2) implies a relation

$$
1=\sum_{i} \Omega_{i}+\Omega_{k}
$$

The parameters $\Omega_{i}, \Omega_{k}$ can be used to rewrite equation (2) at a general time

$$
\frac{1}{a} \frac{d a}{d t}=H_{0}\left(\sum_{i} \Omega_{i} a^{-3\left(1+\omega_{i}\right)}+\Omega_{k} a^{-2}\right)^{1 / 2} .
$$

\section{THE AGE OF THE UNIVERSE AND DISTANCE MEASURES}

Equation (5) can be easily used to calculate the age of the universe. The age is the 


$$
H_{0} t_{0}=\int_{0}^{1} \frac{d a}{a\left[\sum_{i} \Omega_{i} a^{-3\left(1+\omega_{i}\right)}+\Omega_{k} a^{-2}\right]^{1 / 2}} .
$$

By a trivial change of the integral variable from $a$ to redshift $z$, the age expression reduces to

$$
H_{0} t_{0}=\int_{0}^{\infty} \frac{d z}{(1+z)\left[\sum_{i} \Omega_{i}(1+z)^{3\left(1+\omega_{i}\right)}+\Omega_{k}(1+z)^{2}\right]^{1 / 2}} .
$$

It has been shown that the natural cosmological distance for the analysis of gravitational lensing statistics is the comoving distance [17]. The distance a light ray travels can be calculated as follows. Light rays follow null geodesics where $d s^{2}=0$ so that $d t^{2}=R_{0}^{2} a^{2} d \chi^{2}$. With Eq.(2) and the relation $a(t)=(1+z)^{-1}$, the comoving distance is

$$
\chi= \begin{cases}\int_{0}^{z} \frac{d z}{\sqrt{\sum_{i} \Omega_{i}(1+z)^{3\left(1+\omega_{i}\right)}}} & (k=0), \\ \left|\Omega_{k}\right|^{1 / 2} \int_{0}^{z} \frac{d z}{\sqrt{\sum_{i} \Omega_{i}(1+z)^{3\left(1+\omega_{i}\right)}+\Omega_{k}(1+z)^{2}}} & (k= \pm 1) .\end{cases}
$$

Most of classical cosmological tests involve the standard candle (e.g. SNeIa) or standard ruler (e.g. radio source). Therefore both luminosity distance $D^{L}$ and angular diameter distance $D^{A}$ are often used. They are related to the comoving distance as 18

$$
D^{L} \equiv R_{0}^{2} f(\chi) / R(t), \quad D^{A} \equiv R(t) f(\chi)
$$

They are simply related to the measurable, the redshift $z$, through Eq.(8) with the following form

$$
D^{L}=(1+z)^{2} D^{A}= \begin{cases}H_{0}^{-1}(1+z) \chi & k=0, \\ H_{0}^{-1}(1+z)\left|\Omega_{k}\right|^{-1 / 2} \sin \chi & k=+1, \\ H_{0}^{-1}(1+z)\left|\Omega_{k}\right|^{-1 / 2} \sinh \chi & k=-1,\end{cases}
$$

Finally, one often needs to calculate volume element of the universe. In order to take into account the cosmic expansion, it is more convenient to use comoving volume than the traditional physical volume. Within the shell $d \chi$ at $\chi, d V$ reads

$$
d V=4 \pi R_{0}^{3} f^{2}(\chi) d \chi=4 \pi H_{0}^{-3} \begin{cases}\chi^{2} d \chi & k=0 \\ \left|\Omega_{k}\right|^{-3 / 2} \sin ^{2} \chi d \chi & k=+1 \\ \left|\Omega_{k}\right|^{-3 / 2} \sinh ^{2} \chi d \chi & k=-1\end{cases}
$$




\section{GRAVITATIONAL LENSING STATISTICAL PROPERTIES OF SINGULAR ISOTHERMAL SPHERES}

\section{A. Optical depth for multiple image}

First of all, we consider the lensing cross-section [19] due to a specific galaxy. Following Ref. [19], we model the mass density profile of the total galaxy matter as the singular isothermal sphere (SIS). The dimensionless cross-section of multiple image for a point source located at $z_{s}$ produced by a single SIS galaxy at $z_{d}$ is 20

$$
\hat{\sigma}=\pi \theta_{E}^{2}, \quad \theta_{E} \equiv 4 \pi \sigma^{2} \frac{D_{d s}^{A}}{D_{s}^{A}}
$$

where $D_{s}^{A}$ and $D_{d s}^{A}$ are the angular diameter distances from the observer to the source and from the lens to the source respectively, $\theta_{E}$ is the angular radius of Einstein ring and $\sigma$ is the velocity dispersion of the lensing galaxy. Using the comoving distance gives

$$
\hat{\sigma}=16 \pi^{3} \sigma^{4}\left[\frac{f\left(\chi_{s}-\chi_{d}\right)}{f\left(\chi_{s}\right)}\right]^{2} .
$$

Now, let's consider the contributions of an ensemble of galaxies having different luminosities and redshifts. The present-day galaxy luminosity function can be described by the Schechter function 21

$$
\phi_{i}(L) d L=\phi_{i}^{*}\left(L / L_{i}^{*}\right)^{-\alpha_{i}} \exp \left(-L / L_{i}^{*}\right) d\left(L / L_{i}^{*}\right)
$$

where $i$ indicates the morphological type of galaxies: $i=(\mathrm{E}, \mathrm{S} 0, \mathrm{~S})$. The above expression can be converted into the velocity dispersion distribution through the empirical formula between the luminosity and the central dispersion of local galaxies $L / L_{i}^{*}=\left(\sigma / \sigma_{i}^{*}\right)^{g_{i}}$. Finally, the optical depth of multiple image by galaxies at redshifts ranging from 0 to $z_{s}$ for the distant sources like quasars at $z_{s}$ is

$$
\tau\left(z_{s}\right)=\left(\sum_{i=E, S 0, S} F_{i}\right) T\left(z_{s}\right),
$$

The parameter $F_{i}$ represents the effectiveness of the $i$-th morphological type of galaxies in $\|19\|$, which reads 


$$
F_{i} \equiv 16 \pi^{3} H_{0}{ }^{-3}\left\langle n_{0 i} \sigma^{4}\right\rangle=16 \pi^{3} H_{0}{ }^{-3} \phi_{i}^{*} \gamma_{i}\left(b_{i} \sigma_{i}^{*}\right)^{4} \int\left(L / L_{*}\right)^{\alpha_{i}+4 / g_{i}} \exp \left(L / L_{*}\right) d L / L_{*},
$$

where $\gamma_{i}$ is the galaxy morphological composition and $b_{i}$ is the velocity bias between the velocity dispersion of stars and of dark matter particles [22]. The above equation can be further written as

$$
F_{i}=16 \pi^{3} H_{0}^{-3} \phi_{i}^{*} \gamma_{i}\left(b_{i} \sigma_{i}^{*}\right)^{4} \Gamma\left(-\alpha_{i}+4 / g_{i}+1\right), \text { if } L \in(0, \infty),
$$

if the integral is performed from 0 to $\infty$. In practice, the galaxy luminosities have the minimum and maximum limits, and, therefore, Eq.(17) is the maximum estimate of $F_{i}$. The $z_{s}$ dependent factor $T\left(z_{s}\right)$ is

$$
T\left(z_{s}\right)=\left(H_{0} R_{0}\right)^{3} \int_{0}^{\chi_{s}}\left[\frac{f\left(\chi_{s}-\chi_{d}\right)}{f\left(\chi_{s}\right)}\right]^{2} f^{2}\left(\chi_{d}\right) d \chi_{d} .
$$

For general FRW cosmologies with various matter and energy components, an analytic expression is found [17]:

$$
T\left(z_{s}\right)= \begin{cases}\frac{\chi_{s}^{3}}{30}, & (k=0), \\ \left|\Omega_{k}\right|^{-3 / 2}\left[\frac{1}{8}\left(1+3 \cot ^{2} \chi_{s}\right) \chi_{s}-\frac{3}{8} \cot \chi_{s}\right], & (k=+1), \\ \left|\Omega_{k}\right|^{-3 / 2}\left[\frac{1}{8}\left(-1+3 \operatorname{coth}^{2} \chi_{s}\right) \chi_{s}-\frac{3}{8} \operatorname{coth} \chi_{s}\right], & (k=-1),\end{cases}
$$

where $\chi_{s}$ can be calculated through Eq.(8).

As pointed out by Kochaneck [22], although the parameters $\left(\phi_{i}^{*}, L_{i}^{*}, \alpha_{i} ; \sigma_{i}^{*}, g_{i} ; \gamma_{i}\right)$ of galaxies are updated due to recent surveys [23] there are still large uncertainties with them. Therefore we treat $F \equiv \sum_{i} F_{i}$ as normalized factor. One may expect to determine both the dark energy amount and its equation of state. It is necessary to consider general flat universe models $\left(\Omega_{m} ; \Omega_{x}, \omega_{x}\right)$ with $\Omega_{m}+\Omega_{x}=1$. Generally, the optical depth of gravitational lensing depends on the amount of dark energy $\Omega_{x}$ as well as its equation of state $\omega_{x}$. The larger the dark energy amount is, the higher the gravitational lensing probability will be; the more negative the dark energy pressure is, the higher the optical depth will be. 


\section{B. Average image separation}

In addition to optical depth, other interesting quantities for lensing statistics are the mean image separation produced by a specified SIS at a given $z_{d}$ with velocity dispersion $\sigma$ and its average over the distributions of lens velocity dispersion and lens redshift. The angular separation of the two images produced by SIS is

$$
\Delta \theta=2 \theta_{E}=8 \pi \sigma^{2} \frac{f\left(\chi_{s}-\chi_{d}\right)}{f\left(\chi_{s}\right)}
$$

which doesn't depend on impact parameter, and hence implies

$$
\overline{\Delta \theta}=\Delta \theta \quad \text { for } \text { SIS }
$$

where $\overline{\Delta \theta}$ is the mean image separation for the SIS lens at $z_{d}$ with velocity dispersion $\sigma$. The average image separation is obtained by

$$
<\overline{\Delta \theta}>=\frac{1}{\tau} \int \overline{\Delta \theta} d \tau
$$

We take into account the lens redshift distribution as well as the lens velocity dispersion distribution. The latter was generally neglected in literatures. Then the average image separation reduces to

$$
<\overline{\Delta \theta}>=\frac{1}{\tau}\left(\sum_{i=E, S 0, S} F_{i}^{\prime}\right) \Theta\left(z_{s}\right)
$$

where

$$
F_{i}^{\prime} \equiv 128 \pi^{4} H_{0}{ }^{-3}\left\langle n_{0 i} \sigma^{6}\right\rangle=128 \pi^{4} H_{0}{ }^{-3} \phi_{i}^{*} \gamma_{i}\left(b_{i} \sigma_{i}^{*}\right)^{6} \int\left(L / L_{*}\right)^{\alpha_{i}+6 / g_{i}} \exp \left(L / L_{*}\right) d L / L_{*}
$$

if the above integral is performed from 0 to $\infty$, it can be further written as

$$
F_{i}^{\prime} \equiv 128 \pi^{4} H_{0}^{-3} \phi_{i}^{*} \gamma_{i}\left(b_{i} \sigma_{i}^{*}\right)^{6} \Gamma\left(-\alpha+6 / g_{i}+1\right), \text { if } L \in(0, \infty)
$$

and the $z_{s}$ dependent factor $\Theta\left(z_{s}\right)$ is

$$
\Theta\left(z_{s}\right) \equiv\left(H_{0} R_{0}\right)^{3} \int_{0}^{\chi_{s}}\left[\frac{f\left(\chi_{s}-\chi_{d}\right)}{f\left(\chi_{s}\right)}\right]^{3} f^{2}\left(\chi_{d}\right) d \chi_{d}
$$


For general FRW cosmologies with various matter and energy components, an analytic expression is found:

$$
\Theta\left(z_{s}\right)= \begin{cases}\frac{\chi_{s}^{3}}{60}, & (k=0), \\ \left|\Omega_{k}\right|^{-3 / 2} \frac{2}{15} \sin ^{-3} \chi_{s}\left[\left(4-3 \sin ^{2} \chi_{s}\right)+\cos \chi_{s}\left(-4+\sin ^{2} \chi_{s}\right)\right], & (k=+1) \\ \left|\Omega_{k}\right|^{-3 / 2} \frac{2}{15} \sinh ^{-3} \chi_{s}\left[\left(-4-3 \sinh ^{2} \chi_{s}\right)+\cosh \chi_{s}\left(4+\sinh ^{2} \chi_{s}\right)\right], & (k=-1),\end{cases}
$$

where $\chi_{s}$ can be calculated through Eq.(8) as before. If we define $\Delta \theta_{*} \equiv \sum_{i} F_{i}^{\prime} / \sum_{i} F_{i}$, which is determined completely by the intrinsic characteristic parameters of galaxies describing its statistical properties, the average image separation then becomes

$$
<\overline{\Delta \theta}>=\Delta \theta_{*} \Theta\left(z_{s}\right) / T\left(z_{s}\right), \quad \Delta \theta_{*} \equiv\left(\sum_{i=E, S 0, S} F_{i}^{\prime}\right) \div\left(\sum_{i=E, S 0, S} F_{i}\right) .
$$

Thus for an open or closed universe, the average image separation for the SIS lens depends on $\chi_{s}$ (or the source redshift), while for a flat universe, it doesn't but takes a constant value $<\overline{\Delta \theta}>=\Delta \theta_{*} / 2$. This fact has been suggested to test directly the curvature of the universe [24].

\section{Probability distribution of image separation}

Another useful quantity of lensing statistics is the probability distribution of image separation, which is reconsidered here for general cosmologies with various matter and energy components. The differential optical depth for multiple imaging a background object like a quasar by lenses at position between $\chi_{d}$ and $\chi_{d}+d \chi_{d}$ and with luminosity from $L$ to $L+d L$ is

$$
d^{2} \tau_{i}=\gamma_{i} \phi_{i}(L) d L \hat{\sigma} R_{0}^{3} f^{2}\left(\chi_{d}\right) d \chi_{d}
$$

By change of the variable from luminosity to angular separation, the differential optical depth becomes 


$$
\begin{aligned}
\frac{d^{2} \tau_{i}}{d \Delta \theta d \chi_{d}}= & \pi^{2}(8 \pi)^{g_{i}\left(\alpha_{i}-1\right) / 2-1} \gamma_{i} g_{i} \phi_{* i}\left(b_{i} \sigma_{* i}\right)^{g_{i}\left(\alpha_{i}-1\right)} S(\Delta \theta) \Delta \theta^{g_{i}\left(1-\alpha_{i}\right) / 2+1} \\
& \cdot \exp \left\{-\left[8 \pi\left(b_{i} \sigma_{* i}\right)^{2}\right]^{-g_{i} / 2}\left[\frac{f\left(\chi_{s}-\chi_{d}\right)}{f\left(\chi_{s}\right)}\right]^{-g_{i} / 2} \Delta \theta^{g_{i} / 2}\right\}\left[\frac{f\left(\chi_{s}-\chi_{d}\right)}{f\left(\chi_{s}\right)}\right]^{g_{i}\left(\alpha_{i}-1\right)} R_{0}^{3} f^{2}\left(\chi_{d}\right)
\end{aligned}
$$

where we have taken into account the angular resolution bias by the function $S(\Delta \theta)$, which varies for different lens observations and surveys [25]. Then, the probability distribution of image separations becomes

$$
\frac{d P}{d \Delta \theta}=\frac{1}{\tau} \int_{0}^{\chi_{s}} \sum_{i}\left(\frac{d^{2} \tau_{i}}{d \Delta \theta d \chi_{d}}\right) d \chi_{d}
$$

which in general case depends on $\chi_{s}$ (or $z_{s}$, the redshift of the source). However for a flat universe, the above integral scales as $\propto \chi_{s}^{3}$. Combining Eq.(15) and 19, one concludes that the probability distribution of image separations is independent of $\chi_{s}$, regardless of the functional form of the angular resolution bias [26]. Hence the cumulative distribution of image separations can be easily calculated from $d P / d \Delta \theta$ as

$$
P(\Delta \theta)=\int_{0}^{\Delta \theta}\left(\frac{d P}{d \Delta \theta^{\prime}}\right) d \Delta \theta^{\prime}
$$

\section{RESULTS AND DISCUSSIONS}

As shown above, it is easy to analytically calculate the optical depth for multiple image (Eq.(15) and (19)), average image separation (Eq.(28), (19) and (27)) and probability distribution of image separations (Eq.(31)), as well as the age of the universe (Eq.(7)) and the luminosity (angular diameter) distance (Eq.(10)), if the matter and energy components in the universe are given in terms of $\left(\Omega_{i}, \omega_{i}\right)$. It is shown that the lensing statistical properties depend sensitively on the dark energy amount and its equation of state and hence provide an independent probe for the dark energy.

In the above lensing calculations, we have assumed that the comoving number density of galaxies is constant. However, this may not hold true for the realistic situation. The influence of galaxv evolution on the lensing statistics should also be taken into account $\| 27$ 
We have considered this effect by using the galaxy merging model proposed by Broadhurst et al. (1992), since the scenario of galaxy merging can account for both the redshift distribution and the number counts of galaxies at optical and near-infrared wavelengths [28]. There are two effects arising from the galaxy merging: The first is that there are more galaxies and hence more lenses in the past. The second is that galaxies are typically less massive in the past and hence less efficient as lenses. As a result of two effects, the total cross-section remains roughly unchanged [29].

Although the evidence for the existence of a nearly uniform component of dark energy with negative pressure in the universe has been consistently provided by several astronomical observations, we at present know a little about it. It needs more cosmologcial constraints on dark energy to determine its amount and nature. The simple formulae we derived here could make the cosmological tests for dark energy component(s) more convenient.

\section{ACKNOWLEDGMENTS}

I thank X. P. Wu for careful reading of the manuscript and helpful discussions. This work was supported by the National Natural Science Foundation of China, under Grant No. 19903002. 


\section{REFERENCES}

[1] C. Lineweaver, Astrophys. J., 505 (1998) L69.

[2] S. Burles, D. Tytler, Astrophys. J., 499 (1998) 699; S. Burles, D. Tytler, Astrophys. J., 507 (1998) 732.

[3] U.G. Briel, J.P. Henry, H. Bohringer, Astron. Astrophys., 259 (1992) L31; S.D.M. White et al., Nature, 366 (1993) 429; J.J. Mohr, B. Mathiesen, A.E. Evrard, Astrophys. J., 517 (1999) 627.

[4] S. Perlmutter et al., Astrophys. J., 517 (1999) 565; A.G. Riess et al., Astron. J., 116 (1998) 1009.

[5] M.S. Turner, astro-ph/9811454.

[6] S. Weinberg, Rev. Mod. Phys. 61 (1989) 1; S. Carroll et al., Ann. Rev. Astron. Astrophys., 30 (1992) 499; L.M. Krauss, M.S. Turner, Gen. Rel. Grav., 27 (1995) 1137; J.P. Ostriker, P.J. Steinhardt, Nature, 377 (1995) 600.

[7] J.D. Cohn, Living with Lambda, astro-ph/9807128; V. Sahni, A. Starobinsky, Int. J. Mod. Phys. D, in press (astro-ph/9904398).

[8] A. Vilenkin, Phys. Rev. Lett. 53 (1984) 1016; R.L. Davis, Phys. Rev. D35 (1987) 3705; M. Kamionkowski, N. Toumbas, Phys. Rev. Lett. 77 (1996) 587.

[9] B. Ratra and P.J.E. Peebles, Phys. Rev. D37 (1988) 3406; J. Frieman et al, Phys. Rev. Lett. 75 (1995) 2077; K. Coble et al, Phys. Rev. D55 (1995) 1851; R. Caldwell et al, Phys. Rev. Lett. 80 (1998) 1582.

[10] P. Garnavich et al., Astrophys. J., 509 (1998) 74.

[11] G. Huey et al., Phys. Rev. D59 (1999) 063005.

[12] M.S. Turner, M. White, Phys. Rev. D56 (1997) R4439. 
[13] S. Perlmutter, M.S. Turner, M. White, Phys. Rev. Lett., 83 (1999) 670.

[14] E.L. Turner, Astrophys. J., 365 (1990) L43; M. Fukugita et al., MNRAS, 246 (1990) 24; L.M. Krauss, M. White, Astrophys. J., 394 (1992) 385; C.S. Kochanek, Astrophys. J., 466 (1996) 638; A.R. Cooray, Astron. Astrophys., 341 (1999) 653; R. Quast, P.Helbig, Astron. Astrophys., 344 (1999) 721; M. Chiba, Y. Yoshii, Astrophys. J., 510 (1999) 42; I. Waga, A.P.M.R. Miceli, Phys.Rev.D59 (1999) 103507; Y. Mellier, Ann. Rev. Astron. Astrophys., 37 (1999) 127; Z.H. Zhu, Mod. Phys. Lett. A15 (2000) 1023; Y.-C.N. Cheng, L.M. Krauss, Int.J.Mod.Phys.A15 (2000) 6971.

[15] X.P. Wu, F. Hammer, MNRAS, 262 (1993) 187; X.P. Wu, S. Mao, Astrophys. J., 463 (1996) 404; T. Hamana, T. Futamase, MNRAS, 286 (1997) L7; M. Bartelmann et al., Astron. Astrophys., 330 (1998) 1.

[16] J.R. Gott, M.G. Park, H.M. Lee, Astrophys. J., 338 (1989) 1; C.S. Kochanek, MNRAS, 261 (1993) 453.

[17] Z.H. Zhu, Astron. Astrophys., 338 (1998) 777; Z.H. Zhu, Mod. Phys. Lett. A15 (2000) 1023.

[18] S. Carroll et al., Ann. Rev. Astron. Astrophys., 30 (1992) 499.

[19] E.L. Turner, J.P. Ostriker, J.R. Gott, Astrophys. J., 284 (1984) 1.

[20] Schneider P., Ehlers J., Falco E. E., 1992, Gravitational Lenses (Springer Verlag, Berlin); X.P. Wu, Fund. Cos. Phys. 17 (1996) 1.

[21] Peebles P. J. E., 1993, Physical Cosmology (Princeton Univ. Press, Princeton).

[22] C.S. Kochanek, Astrophys. J., 466 (1996) 638.

[23] J. Loveday, B.A. Peterson, G. Efstathiou, S.J. Maddox, Astrophys. J., 390 (1992) 338;

R.O. Marzke, M.J. Geller, J.P. Huchra, H.G. Corvin, Astro. J., 108 (1994) 437.

[24] M.G. Park, J.R. Gott, ApJ 489 (1997) 476; P. Helbig, MNRAS 298 (1998) 395. 
[25] A.O. Jaunsen, M. Jablonski, B.R. Pettersen, R. Stabell, Astron. Astrophys., 300 (1995) 323.

[26] J.R. Gott, M.G. Park, H.M. Lee, Astrophys. J., 338 (1989) 1; M.G. Park, 1996, astro$\mathrm{ph} / 9609204$.

[27] S. Mao, C.S. Kochanek, MNRAS, 268 (1994) 569; D. Jain, N. Panchapakesan, S. Mahajan, V.B. Bhatia, Mod.Phys.Lett.A15 (2000) 41.

[28] T. Broadhurst et al., Nature, 355 (1992) 55.

[29] Z.H. Zhu, X.P. Wu, Astron. Astrophys., 326 (1997) L9. 